\title{
Infection and cancer: biology, therapeutics and prevention
}

\section{Brigette BY Ma \& Anthony TC Chan ${ }^{\dagger}$}

${ }^{\dagger}$ Author for correspondence: Department of Clinical Oncology, Prince of Wales Hospital, Ngan Shing Street, Shatin, New Territories, Hong Kong S.A.R., China m Tel.: +85226 322118 m Fax: +85 226487097

m. brigette@clo.cuhk.edu.hk

\section{Infection and Cancer: Biology, Therapeutics and Prevention}

Hong Kong Cancer Institute and the American Association of Cancer Research in conjunction with the State Key Laboratory in Oncology in South China, The Chinese University of Hong Kong, Hong Kong SAR, China, December 5-7, 2008

The Infection and Cancer: Biology, Therapeutics and Prevention international conference on infection-associated cancer was jointly held by the American Association of Cancer Research (AACR) and the Chinese University of Hong Kong on December 5-7 2009. The 3-day scientific program comprehensively covered $12 \mathrm{key}$ areas concerning the pathobiology and clinical translation of research into cancers associated with Helicobacter pylori (HP), human papillomavirus (HPV), Hepatitis B (HBV) and C (HCV) and the Epstein-Barr virus (EBV). Special focus was given to Asia-prevalent cancers such as hepatocellular carcinoma (HCC), gastric cancer, cervical cancer and nasopharyngeal carcinoma (NPC). The meeting provided an excellent platform for academic exchange between the speakers and the participants, who were primarily basic and clinician scientists representing over 17 countries. This report will focus on the latest insights into the clinical aspect of research in infection-associated cancers.

\section{Primary prevention with population- based vaccines is a promising strategy}

Both HCC and cervical cancers are amongst the top ten most common causes of cancer-related death in the world. Population-based HBV vaccination was first introduced in Northeast Asia where the HBV carriage rate has been reported as up to $20 \%$ in the community. Ding-Shinn Chen (National Taiwan University Hospital, Taipei, Taiwan) reviewed the progress of a nationwide $\mathrm{HBV}$ vaccination program introduced in Taiwan in 1984, which initially targeted newborns and was later extended to all age groups. Over the last 20 years, there has been an encouraging decline in the incidence of HCC in children and teenagers who were vaccinated. Given the long latency of progression from acquiring infection to the development of cirrhosis and HCC, Chen projected that a fall in the incidence of HCC in the adult population should be apparent by the year 2040 .
The discovery of HPV as a cause of cervical cancer by Harold zur Hausen (German Cancer Research Centre, Heidelberg, Germany) is a major breakthrough that made the use of preventive or therapeutic HPV vaccines against this cancer possible. Although the currently available vaccines, Gardasil ${ }^{\mathrm{TM}}$ (Merck Sharp \& Dohme, NJ, USA) and Ceravix ${ }^{\text {тм }}$ (GlaxoSmithKline, Middlesex, UK) give excellent protection against the most common HPV subtypes (16 and 18), they are relatively expensive and ineffective against other subtypes, which account for $30 \%$ of cervical cancers. TC Wu (Johns Hopkins Medical Institutions, Baltimore, MD, USA) anticipated that it may take at least 20 years before the impact of population-based vaccination on the incidence of HCC becomes apparent. Douglas R Lowry (National Cancer Institute - CCR, MD, USA) has developed a broad-spectrum vaccine against the L2 minor capsid protein, which has not yet been tested in humans although clinical trials are being planned. Using a rodent cervix-vaginal challenge, he and his team have demonstrated raised the importance of the cervix's mucosal integrity as a factor affecting the efficacy of a L2 vaccine.

\section{Early detection is important in curbing infection-associated cancers}

An important area of research is the population-based screening of NPC, HCC and gastric cancer in Asia. EBV serology has been used in population-based screening of high-risk groups in endemic regions of NPC; however, its clinical utility is affected by its relatively low specificity. YM Dennis Lo (Chinese University of Hong Kong, China) and his team pioneered the technique of quantitative PCR in measuring the level of plasma EBV DNA as a biomarker in NPC. This marker is currently being evaluated as a tool for mass screening of NPC in Hong Kong. He reported the result of a pilot study, where
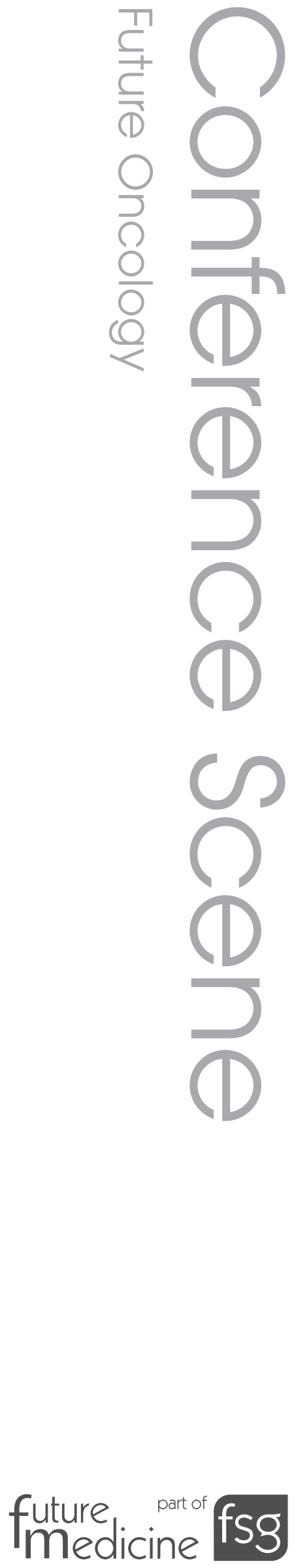
over 1060 out of 1180 asymptomatic recruited subjects underwent plasma EBV DNA analysis. Nine cases $(0.8 \%)$ had persistently positive levels after a period of 2-3 weeks, of whom two cases of early-stage NPC were diagnosed. Interestingly, one of these cases had undetectable EBV VCA-IgA level at diagnosis. Based on this promising result, a larger screening study is being planned.

The screening of asymptomatic carriers of $\mathrm{HBV}$ or $\mathrm{HCV}$ infection with serial sampling of serum $\alpha$-fetoprotein (AFP) and/or abdominal ultrasound examinations (US) has enabled the early detection of HCC, at a rate of 2.2-13.2\% as reported in several cohort studies. Tony Mok (Chinese University of Hong Kong) discussed controversies over the optimal frequency of screening. He and his colleagues evaluated the feasibility of an intensive screening program in Hong Kong, where asymptomatic HBV carriers with baseline abnormalities on abdominal US and/or AFP levels would undergo hepatic angiogram and lipiodol-computed tomography scan to exclude HCC. This was followed by 3-monthly AFP and abdominal US examinations. Over 1000 subjects were recruited, of whom 171 were eligible for intensive screening and the rest underwent the 'conventional' 6-monthly screening. The HCC detection rate was $27.5 \%$ in the intensively screened group compared with $1.1 \%$ in the other. Mok stressed that a higher detection rate does not always translate into a higher resection rate and, therefore, for screening to achieve its ultimate goal of lowering HCC-specific mortality, more effective treatment is needed.

At least three nonrandomized and randomized Japanese studies totaling approximately 3000 subjects have demonstrated that HP eradication is associated with reduced risk of gastric cancer. Junichi Akiyama (International Medical Center of Japan, Tokyo, Japan) and colleagues have developed a system of screening high-risk groups for HP eradication. This involves the use of novel endoscopic imaging systems such as autofluorescence and narrow band imaging for the detection of premalignant gastric lesions. Another less invasive method of identifying high-risk people with HP serology and assessment of pepsinogen I/II status have been evaluated in large-scale population studies in Japan. In one study involving over 6900 participants, those with elevation of pepsinogen levels and/or HP antibodies had the highest annual incidence of $\mathrm{GC}(0.36$ to $0.6 \%)$ than those without.

\section{Anticancer therapies targeting the infective organism are effective against some cancers}

The presence of heavy viral load as shown by a persistently high level of plasma HBV DNA is an important risk factor of HCC in HBV carriers. In a meta-analysis by Joseph Sung (Chinese University of Hong Kong) and team, the use of lamivudine was associated with a lowered risk of developing HCC by $72 \%$ in five prospective studies (2289 subjects), while the use of interferon was associated with a $34 \%$ reduction in responding patients in 12 prospective studies (2742 subjects).

Immunotherapy against EBV-associated lymphoproliferative disease was addressed by Cliona M Rooney (Baylor College of Medicine, TX, USA). Using cytotoxic T-lymphocyte (CTL) therapy specific to LMP1 and LMP2, she and her colleagues were able to demonstrate clinical activity in patients with recurrent lymphomas such as natural killer (NK) cell lymphoma and Hodgkin's disease. Prolonged disease remissions were also observed in some patients who have completed chemotherapy or bone marrow transplantation. These clinical findings were accompanied by the detection of host immune response and fall in the levels of plasma EBV DNA in some patients.

Another approach to targeting the EBV is to induce EBV lytic antigen expression pharmacologically in EBV-associated cancers such as NPC, where EBV latent infection plays an important role in pathogenesis. Qian Tao (Chinese University of Hong Kong) and colleagues have been able to induce re-expression of EBV-latent proteins (Zta) and also partial reversal of methylated EBV promoters in patients with treatment-refractory NPC, who were treated with the demethylating agent azacitidine. An ongoing study of azacitidine and a histone deacetylase inhibitor is being conducted in locally recurrent NPC.

The development of therapeutic vaccines for HPV-associated cervical cancer was the focus of CJM Melief's (Leiden University Medical Center, the Netherlands) talk, whose team has designed a synthetic long-peptide-based vaccine specific to the E6 and E7 proteins. In early clinical trials of this agent, encouraging clinical and immunological responses have been reported in some patients with HPV-associated vulval intraepithelial neoplasia treated with this vaccine after 12 months of therapy. Studies in cervical intraepithelial neoplasia and advanced cervical cancers are now underway. 


\section{Summary}

This meeting has provided a timely overview of the recent advances and ongoing challenges in the translational research of infection-associated cancers. The development of primary preventive vaccines and therapeutic vaccines for cervical cancers, and immunotherapy against EBVassociated cancers have shown great promise and warrant further evaluation. Novel populationbased screening strategies should be evaluated prospectively for NPC and gastric cancer.

\section{Acknowledgements}

We would like to acknowledge the American Association of Cancer Research for their support in this conference, especially Waun Ki Hong (MD Anderson Cancer Center, TX, USA) whose advice has been invaluable.

\section{Financial \& competing interests disclosure}

The authors have no relevant affiliations or financial involvement with any organization or entity with a financial interest in or financial conflict with the subject matter or materials discussed in the manuscript. This includes employment, consultancies, honoraria, stock ownership or options, expert testimony, grants or patents received or pending, or royalties.

No writing assistance was utilized in the production of this manuscript.

\section{Highlights}

- Primary prevention with population-based vaccination is a promising strategy against cervical cancer and hepatitis-B infection-related liver cancer.

- Early detection is important in curbing infection-associated cancers.

- Therapeutic strategies that are directed against the infective organism are effective against some cancers.

\section{Affiliations}

\section{- Brigette BY Ma}

Associate Professor, State Key Laboratory in Oncology in South China, Sir YK Pao Centre for Cancer, Department of Clinical Oncology, Hong Kong Cancer Institute \& Li Ka Shing Institute of Health Sciences, Chinese University of Hong Kong; Department of Clinical Oncology, Prince of Wales Hospital, Ngan Shing Street, Shatin, New Territories, Hong Kong SAR, China Tel.: +85226322 118

Fax: +85226487097

brigette@clo.cuhk.edu.hk

\section{Anthony TC Chan}

State Key Laboratory in Oncology in South China, Sir YK Pao Centre for Cancer, Department of Clinical Oncology, Hong Kong Cancer Institute \& Li Ka Shing Institute of Health Sciences, Chinese University of Hong Kong

Tel.: +85226322 119

Fax: +85226487097 